\title{
Pacific
}

Journal of

Mathematics

\section{SUBALGEBRAS OF LITTLE LIPSCHITZ ALGEBRAS}

\author{
NiKolai IsAaC WeAVER
}

Volume 173 No. 1

March 1996 


\title{
SUBALGEBRAS OF LITTLE LIPSCHITZ ALGEBRAS
}

\author{
NIK WEAVER
}

We present a version of the Stone-Weierstrass theorem for $\operatorname{lip}(X)$ and use it to characterize the closed subalgebras of $\operatorname{lip}(X)$ which contain the constant functions.

Let $X$ be a compact metric space. Then the Lipschitz space $\operatorname{Lip}(X)$ is the Banach space of all real-valued Lipschitz functions on $X$ with the norm

$$
\|f\|_{L}=\max \left(\|f\|_{\infty}, L(f)\right)
$$

where

$$
L(f)=\sup _{\substack{x, y \in X \\ x \neq y}} \frac{|f(x)-f(y)|}{\rho(x, y)}
$$

is the Lipschitz number of $f$.

Let $\widehat{X}$ be the disjoint union of $X$ (as a topological space) and the space

$$
X^{2}-\{(x, x): x \in X\}
$$

and define a linear map $\Phi: \operatorname{Lip}(X) \rightarrow C_{b}(\hat{X})$ (= the bounded continuous real-valued functions on $\widehat{X}$ ) by $\Phi f(x)=f(x)$ and

$$
\Phi f(x, y)=\frac{f(x)-f(y)}{\rho(x, y)} .
$$

This map is an isometry. The little Lipschitz space $\operatorname{lip}(X)$ is then defined to be the subspace of $\operatorname{Lip}(X)$ consisting of those functions $f$ with the property that for every $\epsilon>0$ there exists $\delta>0$ such that $\rho(x, y) \leq \delta$ implies $|\Phi f(x, y)| \leq \epsilon$. It is easy to check that $\operatorname{lip}(X)=\Phi^{-1}\left(C_{0}(\widehat{X})\right)$.

All of our results hold for complex as well as real scalars, provided that in the complex case one assumes all subalgebras under discussion are selfadjoint (i.e. if $f \in \mathcal{A}$ then $\bar{f} \in \mathcal{A}$ ). Thus the restriction to real scalars is simply a convenience.

The purpose of this paper is to investigate the structure of closed subalgebras of $\operatorname{lip}(X)$ which contain the constant functions. It is known ([Sh], p. 249; [He], Corollaries 1 and 2) that there can exist such subalgebras which separate points but are not equal to $\operatorname{lip}(X)$, so that a naive version of the 
Stone-Weierstrass theorem fails to hold. However, Hedberg ([He], Theorem 1) showed that for a certain type of metric space $X$ a sort of "locally uniform" separation property suffices to imply the desired conclusion. We discuss this result, and related results due to Bade-Curtis-Dales and Hanin, following Theorem 1.4 below.

The Stone-Weierstrass theorem allows one to easily characterize the closed subalgebras of $C(X)$ for compact Hausdorff spaces $X$ ([St], Theorem 5 ): for any closed subalgebra $\mathcal{A}$ of $C(X)$ which contains the constant functions, there is an equivalence relation on $X$ such that $\mathcal{A}$ is naturally isometrically isomorphic to $C(Y)$, where $Y$ is the topological quotient. In contrast, using the "pseudo" Stone-Weierstrass theorem to determine the structure of subalgebras of $\operatorname{lip}(X)$ is surprisingly nontrivial. This result (Theorem 3.4, the main theorem of this paper) states that for every closed subalgebra $\mathcal{A}$ of $\operatorname{lip}(X)$ which contains the constant functions, there exists a nonexpansive image $Y$ of $X$ such that $\mathcal{A}$ is naturally isometrically isomorphic to $\operatorname{lip}(Y)$. (See the start of $\S 3$ for the exact definition of $Y$.)

I wish to thank the referee for suggesting a way to simplify the exposition of $\S 1$ as well as several other improvements. This material is based upon work supported under a National Science Foundation graduate fellowship.

\section{The pseudo Stone-Weierstrass theorem.}

In this section we present a version of the Stone-Weierstrass theorem for little Lipschitz algebras (Theorem 1.5). Most of the results of this section have already appeared elsewhere in some form; furthermore, our pseudo Stone-Weierstrass theorem is closely related to results in $[\mathbf{B C D}]$ and $[\mathbf{H e}]$. Thus no great novelty is claimed here. However, from the point of view of applications, we feel that our version of the result is the simplest to use; we give an example to illustrate this point at the end of the section. We also use it in $\S 3$.

The following proposition is standard (e.g. see [Sh], §I.1).

Proposition 1.1. Let $X$ be a compact metric space. Then $\operatorname{lip}(X)$ is a closed subalgebra of $\operatorname{Lip}(X)$ which contains the constant functions.

We write $\mathcal{A} \leq \operatorname{lip}(X)$ to indicate that $\mathcal{A}$ is a closed subalgebra of $\operatorname{lip}(X)$ which contains the constant functions. The next proposition is essentially ([He], Lemma 2). (That result is stated for the case where the metric of. $X$ is of the form $\rho^{\alpha}$ for some metric $\rho$ and $\alpha \in(0,1)$; but the proof is also valid for $\alpha=1$, i.e. the general case.)

Proposition 1.2. Let $X$ be a compact metric space and let $\mathcal{A} \leq \operatorname{lip}(X)$. Then $\mathcal{A}$ is a sublattice of $\operatorname{Lip}(X)$. 
A proof of Proposition 1.2 which is possibly simpler than that given in $[\mathbf{H e}]$ goes as follows. Note first that since $f \vee g=(f+g+|f-g|) / 2$ and $f \wedge g=(f+g-|f-g|) / 2$, it suffices to show that $|f| \in \mathcal{A}$ whenever $f \in \mathcal{A}$. Wlog we may assume that $\|f\|_{L} \leq 1$. Now the derivative $g$ of the function $G: x \mapsto|x|$ on $[-1,1]$ is a step function; approximate it in $L^{1}$ norm by a polynomial $h$ such that $\|h\|_{\infty} \leq 1$. Let $H=\int h$ and observe that $H$ is a polynomial, hence $H \circ f \in \mathcal{A}$, and that $\|G-H\|_{\infty}$ is small. Finally show that $H \circ f$ approximates $G \circ f=|f|$ in Lipschitz norm, hence $|f| \in \mathcal{A}$. (In the last step, use the fact that $(G-H) \circ f$ has small Lipschitz number, since $\|G-H\|_{\infty}$ is small and $f \in \operatorname{lip}(X)$.)

We mentioned in the introduction that to get a Stone-Weierstrass type theorem we have to set some kind of uniform separation condition on $\mathcal{A}$. The relevant condition is the following:

there exists $a>1$ such that for any $x, y \in X$, some $f \in \mathcal{A}$ satisfies $\|f\|_{L} \leq a$ and $|f(x)-f(y)|=\rho(x, y)$.

We say that $\mathcal{A}$ has the separation property if it satisfies this condition.

The next result gives an equivalent formulation of the separation property. A more general form of this result appears as Proposition 3.1 of [W1]. Note that the proposition relies on the fact that joins and meets do not increase the norm, i.e.

$$
\|f \vee g\|_{L} \leq \max \left(\|f\|_{L},\|g\|_{L}\right)
$$

and similarly for $f \wedge g$. (See [W2] for a full discussion of the rich interplay between lattice structure and norm in Lipschitz spaces.)

Proposition 1.3. Let $X$ be a compact metric space and let $\mathcal{A} \leq \operatorname{lip}(X)$. Then $\mathcal{A}$ has the separation property if and only if there exists $b>1$ such that for any $g \in \operatorname{Lip}(X)$ and finite subset $S \subset X$, some $f \in \mathcal{A}$ satisfies $\|f\|_{L} \leq b \cdot\|g\|_{L}$ and $\left.f\right|_{S}=\left.g\right|_{S}$.

Proof. $\Rightarrow$ ) Suppose $\mathcal{A}$ has the separation property for some $a>1$ and let $S \subset X$ be a finite subset and $g \in \operatorname{Lip}(X)$. Wlog suppose $\|g\|_{L}=1$. Then for each pair of distinct elements $x, y \in S$ we can use the separation property to find a function $f \in \mathcal{A}$ such that $\|f\|_{L} \leq a$ and $|f(x)-f(y)|=$ $|g(x)-g(y)|$, since the latter is $\leq \rho(x, y)$. Multiplying $f$ by \pm 1 we may assume $f(x)-f(y)=g(x)-g(y)$. Now define

$$
f_{x y}(z)=((f(z)+g(x)-f(x)) \wedge 1) \vee-1 .
$$

In other words, shift $f$ so that it agrees with $g$ at $x$ and $y$, and then truncate at \pm 1 . Then $f_{x y}$ belongs to $\mathcal{A}$ by Proposition 1.2 and satisfies $f_{x y}(x)=g(x)$, $f_{x y}(y)=g(y)$, and $\left\|f_{x y}\right\|_{L} \leq a$. Finally let

$$
f=\bigvee_{x \in S} \bigwedge_{\substack{y \in S \\ y \neq x}} f_{x y}
$$


this also belongs to $\mathcal{A}$ by Proposition 1.2, it agrees with $g$ on $S$, and it satisfies $\|f\|_{L} \leq a=a \cdot\|g\|_{L}$.

$\Leftarrow)$ Suppose that the condition given in the statement of the proposition holds and let $x, y \in X$. Extend the function $x \mapsto \rho(x, y) / 2, y \mapsto-\rho(x, y) / 2$ to a function $g \in \operatorname{Lip}(X)$ with $\|g\|_{L} \leq \max (1, \rho(x, y) / 2$ ) (see Theorem 1 of [Mc] for the required extension theorem). Then apply the assumed condition with $S=\{x, y\}$; we get $f \in \mathcal{A}$ such that $|f(x)-f(y)|=|g(x)-g(y)|=\rho(x, y)$ and

$$
\|f\|_{L} \leq b \cdot\|g\|_{L} \leq b \cdot \max (1, \Delta(X) / 2),
$$

where $\Delta(X)$ denotes the diameter of $X$. As the right side is independent of $x$ and $y$, this completes the proof.

By Theorem 3.6 of $[\mathbf{B C D}]$, if the metric of $X$ is of the form $\rho^{\alpha}$ and $\mathcal{A}$ is a closed subspace of $\operatorname{lip}(X)$ which satisfies the condition in Proposition 1.3 , then $\mathcal{A}=\operatorname{lip}(X)$. This result easily generalizes to arbitrary compact metrics ([Ha], Theorem 3); from the general result and Proposition 1.3 we immediately obtain the following theorem.

Theorem 1.4 (pseudo Stone-Weierstrass). Let $X$ be a compact metric space and suppose $\mathcal{A} \leq \operatorname{lip}(X)$ has the separation property. Then $\mathcal{A}=\operatorname{lip}(X)$.

This theorem is only meaningful in the case that $\operatorname{lip}(X)$ itself has the separation property. In general the right condition on $\mathcal{A}$ is that there should exist a constant $c>1$ such that for any $x, y \in X$ and any $g \in \operatorname{lip}(X)$, some $f \in \mathcal{A}$ satisfies $\|f\|_{L} \leq c \cdot\|g\|_{L}$ and $|f(p)-f(q)|=|g(p)-g(q)|$. If $\mathcal{A} \leq \operatorname{lip}(X)$ has this property then $\mathcal{A}=\operatorname{lip}(X)$; one can prove this by arguments similar to those used to prove Theorem 1.4 or else as an easy corollary to Theorem 3.4 below.

We have already seen the close relation between Theorem 1.4 and results of $[\mathbf{B C D}]$. In addition, Hedberg ([He], Theorem 1) proved a related theorem in the case that $X$ has a metric of the form $\rho^{\alpha}$ for some metric $\rho$ and $\alpha \in$ $(0,1)$. There our separation property was replaced by a more complicated but equivalent "local" version, which can be formulated as follows:

$\mathcal{A}$ separates points, and for every $x \in X$ there exist numbers $M_{x}, \delta_{x}>0$ such that for every $y$ within $\delta_{x}$ of $x$ there exists $f \in \mathcal{A}$ with $|f(x)-f(y)|=\rho(x, y)$ and such that $|\Phi f(w, z)| \leq M_{x}$ for all $w, z$ within the ball of radius $\rho(x, y)$ about $x$.

It is clear that our global property implies the local property: just take $M_{x}=a$ for all $x$ and choose $\delta_{x}$ arbitrarily. For the converse, suppose the global property fails and for each $n$ choose $x_{n}, y_{n} \in X$ such that for $f \in \mathcal{A}$, $\left|f\left(x_{n}\right)-f\left(y_{n}\right)\right|=\rho\left(x_{n}, y_{n}\right)$ implies $\|f\|_{L} \geq n$. Let $(x, y)$ be a cluster point of the sequence $\left(x_{n}, y_{n}\right) \subset X \times X$; it is then easy to see that if $x \neq y$ 
then the existence of $f \in \mathcal{A}$ such that $f(x) \neq f(y)$ leads to a contradiction, while $x=y$ more directly contradicts the local property. Thus Hedberg's separation property is equivalent to ours, as claimed.

According to $([\mathbf{H a}]$, Theorem 1$), \operatorname{lip}(X)^{* *}$ is isometrically isomorphic to $\operatorname{Lip}(X)$ by the natural map if and only if $\operatorname{lip}(X)$ satisfies the condition given in Proposition 1.3 for all $b>1$. The forward direction is a simple application of weak* density of the unit ball (Goldstine's theorem), and one can similarly draw the conclusion that $\operatorname{lip}(X)$ has the separation property for all $a>1$. Interestingly, however, by an obvious generalization of ([BCD], Theorem $3.5)$ one can show that the reverse direction of Hanin's theorem only requires satisfaction for some $b>1$ (or equivalently, for some $a>1$ ). Thus, in general, satisfaction for some constant implies satisfaction for all constants and is equivalent to the above duality statement.

In the case of complex scalars the argument of the preceding paragraph carries over without alteration. This circumstance is somewhat mysterious since typically one is only able to directly check satisfaction for $b>\sqrt{2}$. Thus, to get the duality result a [BCD]-type argument is needed in the complex case even though the much simpler argument of $[\mathbf{H a}]$ suffices for the real case.

To illustrate the utility of the pseudo Stone-Weierstrass theorem, we now use it to give a quick proof of the important result ([BCD], Corollary 3.7).

Corollary 1.5. Let $X$ be a compact metric space and let $\alpha \in(0,1)$. Let $X^{\alpha}$ be the set $X$ together with the metric $\rho^{\alpha}$. Then $\operatorname{Lip}(X)$ is dense in $\operatorname{lip}\left(X^{\alpha}\right)$.

Proof. The fact that $\operatorname{Lip}(X) \subset \operatorname{lip}\left(X^{\alpha}\right)$ is well-known and easy. We show that the closure of $\operatorname{Lip}(X)$ in $\operatorname{lip}\left(X^{\alpha}\right)$ has the separation property with $a=$ $2 \cdot \max \left(1, \Delta\left(X^{\alpha}\right)\right)$, where $\Delta\left(X^{\alpha}\right)$ denotes the diameter of $X^{\alpha}$. Fix $x, y \in X$ and define

$$
f(z)=2\left(\rho^{\alpha}(x, z)-\rho^{\alpha}(x, y) / 2\right) \vee 0 .
$$

Then the norm of $f$ as an element of $\operatorname{Lip}\left(X^{\alpha}\right)$ is $\leq a$ and $|f(x)-f(y)|=$ $\rho^{\alpha}(x, y)$.

To show $f \in \operatorname{Lip}(X)$, define $g \in \operatorname{Lip}(X)$ by $g(z)=\rho(x, z)$ and $h: \mathbf{R} \rightarrow \mathbf{R}$ by

$$
h(t)=2\left(t^{\alpha}-\rho^{\alpha}(x, y)\right) \vee 0 .
$$

Then $h$ is Lipschitz on the interval $\left[0, \Delta\left(X^{\alpha}\right)\right]$ since it is piecewise differentiable with bounded derivative. Since $f=h \circ g$ this implies that $f \in \operatorname{Lip}(X)$. as desired.

Note that Corollary 1.5 implies that $\operatorname{lip}\left(X^{\alpha}\right)$ has the separation property ([BCD], Lemma 3.3). 


\section{Quotients of metric spaces.}

In this section we collect some fairly trivial material on quotients of metric spaces. Here we do not require compactness.

Let $\sim$ be an equivalence relation on a metric space $X$. We shall now describe a "quotient" metric space with a certain universal property. It will be constructed by first defining a "quotient pseudo-metric" on $X$, then identifying elements to get a metric space. We need the following terminology: for $f: X \rightarrow Y$ and any pseudo-metric $\rho^{\prime}$ on $X$, we say that $f$ (resp. $\rho^{\prime}$ ) respects $\sim$ if $x \sim y$ implies $f(x)=f(y)$ (resp. $\rho^{\prime}(x, y)=0$ ).

Our first candidate for a quotient pseudo-metric is defined as follows. Let $P_{X}$ be the set of all pseudo-metrics $\rho^{\prime}$ on $X$ which respect $\sim$ and which satisfy $\rho^{\prime}(x, y) \leq \rho(x, y)$ for all $x, y \in X$. (As usual, $\rho$ is the original metric on $X$.) Then define the pseudo-metric $\tilde{\rho}_{1}$ by

$$
\tilde{\rho}_{1}(x, y)=\sup \left\{\rho^{\prime}(x, y): \rho^{\prime} \in P_{X}\right\} .
$$

Similarly, let $F_{X}$ be the set of all nonexpansive functions $f: X \rightarrow \mathbf{R}$ which respect $\sim$, and define another possible quotient pseudo-metric $\tilde{\rho}_{2}$ by

$$
\tilde{\rho}_{2}(x, y)=\sup \left\{|f(x)-f(y)|: f \in F_{X}\right\} .
$$

Finally, we have the following third candidate. For $x, y \in X$ let a chain from $x$ to $y$ be a finite sequence $x_{1}, y_{1}, \ldots, x_{n}, y_{n}$ of elements of $X$ such that $x_{1} \sim x, y_{n} \sim y$, and $y_{i} \sim x_{i+1}$ for $1 \leq i<n$. Let $C_{x y}$ be the set of all chains from $x$ to $y$. Then define $\tilde{\rho}_{3}$ by

$$
\tilde{\rho}_{3}(x, y)=\inf \left\{\rho\left(x_{1}, y_{1}\right)+\ldots+\rho\left(x_{n}, y_{n}\right):\left(x_{1}, y_{1}, \ldots, x_{n}, y_{n}\right) \in C_{x y}\right\} .
$$

We call the number $\rho\left(x_{1}, y_{1}\right)+\ldots+\rho\left(x_{n}, y_{n}\right)$ the length of the chain.

It is fairly easy to see that $\tilde{\rho}_{1}, \tilde{\rho}_{2}$, and $\tilde{\rho}_{3}$ are pseudo-metrics on $X$ which respect $\sim$; we leave this task to the reader. In fact, they are all actually the same pseudo-metric.

Proposition 2.1. Let $X$ be a metric space and let $\sim$ be an equivalence relation on $X$. Then the pseudo-metrics $\tilde{\rho}_{1}, \tilde{\rho}_{2}$, and $\tilde{\rho}_{3}$ defined above are all equal.

Proof. Let $x, y \in X$.

$\tilde{\rho}_{1}(x, y) \leq \tilde{\rho}_{2}(x, y)$ : For any $\rho^{\prime} \in P_{X}$, the function $f: X \rightarrow \mathbf{R}$ defined by $f(x)=\rho^{\prime}(x, y)$ belongs to $F_{X}$ and satisfies

$$
|f(x)-f(y)|=\rho^{\prime}(x, y) .
$$

Hence every number in the sup which defines $\tilde{\rho}_{1}(x, y)$ is also in the sup which defines $\tilde{\rho}_{2}(x, y)$, so $\tilde{\rho}_{1}(x, y) \leq \tilde{\rho}_{2}(x, y)$. 
$\tilde{\rho}_{2}(x, y) \leq \tilde{\rho}_{3}(x, y):$ Let $f \in F_{X}$ and let $x_{1}, y_{1}, \ldots, x_{n}, y_{n}$ be a chain from $x$ to $y$. Then since $f$ respects $\sim$ we have $f(x)=f\left(x_{1}\right), f(y)=f\left(y_{n}\right)$, and $f\left(y_{i}\right)=f\left(x_{i+1}\right)$ for $1 \leq i<n$. So

$$
\begin{aligned}
|f(x)-f(y)| & \leq\left|f\left(x_{1}\right)-f\left(y_{1}\right)\right|+\ldots+\left|f\left(x_{n}\right)-f\left(y_{n}\right)\right| \\
& \leq \rho\left(x_{1}, y_{1}\right)+\ldots+\rho\left(x_{n}, y_{n}\right) .
\end{aligned}
$$

(The last inequality holds because $f$ is nonexpansive.) Thus every number in the sup which defines $\tilde{\rho}_{2}(x, y)$ is $\leq$ every number in the inf which defines $\tilde{\rho}_{3}(x, y)$, so $\tilde{\rho}_{2}(x, y) \leq \tilde{\rho}_{3}(x, y)$.

$\tilde{\rho}_{3}(x, y) \leq \tilde{\rho}_{1}(x, y)$ : We know that $\tilde{\rho}_{3}$ is a pseudo-metric on $X$ that respects $\sim$, and it is clear that $\tilde{\rho}_{3} \in P_{X}$. Thus $\tilde{\rho}_{3}(x, y)$ belongs to the sup which defines $\tilde{\rho}_{1}(x, y)$, so that $\tilde{\rho}_{3}(x, y) \leq \tilde{\rho}_{1}(x, y)$ as desired. This completes the proof.

Let $\tilde{X}$ be the set $X$ modulo the equivalence relation which sets two elements equal if their $\tilde{\rho}_{1}$-distance is zero. Note that this equivalence relation may be strictly stronger than $\sim$; this certainly happens if $\sim$ is not closed (i.e. not a closed subset of $X \times X$ ), but, as the reader may check, there also exist counterexamples even when $\sim$ is closed and $X$ is compact.

The pseudo-metric $\tilde{\rho}_{1}=\tilde{\rho}_{2}=\tilde{\rho}_{3}$ defines a metric on $\tilde{X}$; we call the resulting metric space the metric quotient of $X$ by $\sim$.

For any map $f$ between metric spaces $(X, \rho)$ and $(Y, \sigma)$, we define $L(f)=$ $\sup \sigma(f(x), f(y)) / \rho(x, y)$. This agrees with the definition we gave earlier of $L(f)$ for maps into $\mathbf{R}$. Now $\widetilde{X}$ has the following universal property.

Proposition 2.2. Let $(X, \rho)$ and $(Y, \sigma)$ be metric spaces, let $\sim$ be an equivalence relation on $X$, and let $\tilde{X}$ be the metric quotient of $X$ by $\sim$. If $f: X \rightarrow Y$ is a Lipschitz function which respects $\sim$, then its lift $\tilde{f}: \tilde{X} \rightarrow Y$ is a Lipschitz function and satisfies $L(\tilde{f})=L(f)$.

Proof. First define a pseudo-metric $\rho^{\prime}$ on $X$ by setting

$$
\rho^{\prime}(x, y)=\sigma(f(x), f(y)) / L(f) .
$$

Then $\rho^{\prime} \in F_{X}$ and this shows that the lifted map $\tilde{f}$ is well-defined.

$L(\tilde{f}) \geq L(f)$ since $\tilde{\rho}_{2}(x, y) \leq \rho(x, y)$ for all $x, y \in X$. The converse inequality will show that $\tilde{f}$ is Lipschitz and complete the proof.

Let $x, y \in X$ and consider the map $g: X \rightarrow \mathbf{R}$ defined by

$$
g(z)=\frac{\sigma(f(y), f(z))}{L(f)} .
$$

Now $g \in F_{X}$, so

$$
\tilde{\rho}_{2}(x, y) \geq|g(x)-g(y)|=\frac{\sigma(f(x), f(y))}{L(f)}
$$


that is, $\sigma(f(x), f(y)) \leq L(f) \cdot \tilde{\rho}_{2}(x, y)$. This shows that $L(\tilde{f}) \leq L(f)$ as desired.

\section{Subalgebras of $\operatorname{lip}(X)$.}

Let $\mathcal{A} \leq \operatorname{lip}(X)$. Then for $x, y \in X$ we define $x \sim y$ if $f(x)=f(y)$ for all $f \in \mathcal{A}$, and we define $X_{\mathcal{A}}$ to be the set $X / \sim$ with metric given by

$$
\rho_{\mathcal{A}}([x],[y])=\sup \left\{|f(x)-f(y)|: f \in \mathcal{A},\|f\|_{L} \leq 1\right\} .
$$

We now aim to show that $\mathcal{A}$ is naturally isometrically isomorphic to $\operatorname{lip}\left(X_{\mathcal{A}}\right)$; we do this in Theorem 3.4.

Note that it is generally not true that $\mathcal{A}$ is naturally isometrically isomorphic to $\operatorname{lip}(\tilde{X})$, where $\tilde{X}$ is the metric quotient by $\sim$. This even fails in the case that $\mathcal{A}$ separates points, i.e. $X=\widetilde{X}$; see ([He], Corollaries 1 and 2$)$ for a method of constructing counterexamples.

We mention that Lip spaces can be defined for noncompact metric spaces and that the following theorem remains true for noncompact $X$.

Lemma 3.1. Let $X$ be a compact metric space and let $\sim$ be an equivalence relation on $X$. Let $\tilde{X}$ be the metric quotient of $X$ by $\sim$ and let $\pi: X \rightarrow \tilde{X}$ be the natural map. Then the map $T_{\pi}: f \mapsto f \circ \pi$ isometrically embeds $\operatorname{Lip}(\tilde{X})$ into $\operatorname{Lip}(X)$.

Proof. Let $f \in \operatorname{Lip}(\tilde{X})$; it is clear that $\|f\|_{\infty}=\left\|T_{\pi} f\right\|_{\infty}$. Since $\pi$ is nonexpansive, $L\left(T_{\pi} f\right) \leq L(f)$. For the converse inequality, let $a=L\left(T_{\pi} f\right)$. Then $L\left(T_{\pi} f / a\right)=1$ and so $T_{\pi} f / a \in F_{X}$; thus for all $x, y \in X$

$$
\tilde{\rho}_{2}(x, y) \geq\left|\left(T_{\pi} f / a\right)(x)-\left(T_{\pi} f / a\right)(y)\right|=|f([x])-f([y])| / a .
$$

That is, $|f([x])-f([y])| \leq a \cdot \tilde{\rho}_{2}(x, y)$. This shows that $L(f) \leq a=L\left(T_{\pi} f\right)$. We conclude that $\|f\|_{L}=\left\|T_{\pi} f\right\|_{L}$, so that $T_{\pi}$ is an isometry.

Lemma 3.2. Let $X, \sim, \tilde{X}, \pi$, and $T_{\pi}$ be as in Lemma 3.1. Then

$$
T_{\pi}(\operatorname{Lip}(\tilde{X})) \cap \operatorname{lip}(X) \subset T_{\pi}(\operatorname{lip}(\tilde{X})) .
$$

Proof. Let $f \in \operatorname{lip}(X)$ and suppose $f=T_{\pi}(g)$ for some $g \in \operatorname{Lip}(\tilde{X})$; this implies that $f$ respects $\sim$ and that $g=\tilde{f}$, the lift of $f$ to $\widetilde{X}$. We will show $\tilde{f} \in \operatorname{lip}(\tilde{X})$. Let $\epsilon>0$.

Find $\delta>0$ such that $\rho(x, y) \leq \delta$ implies $|f(x)-f(y)| \leq \epsilon \cdot \rho(x, y)$, and choose $x, y \in X$ such that $\tilde{\rho}_{3}(x, y)<\delta$. For any $a \in \mathbf{R}$ such that 
$\tilde{\rho}_{3}(x, y)<a<\delta$, find a chain $x_{1}, y_{1}, \ldots, x_{n}, y_{n}$ from $x$ to $y$ whose length is $\leq a$. Then $\rho\left(x_{i}, y_{i}\right) \leq a<\delta$ for $1 \leq i \leq n$, hence

$$
\begin{aligned}
|\tilde{f}([x])-\tilde{f}([y])| & \leq\left|f\left(x_{1}\right)-f\left(y_{1}\right)\right|+\ldots+\left|f\left(x_{n}\right)-f\left(y_{n}\right)\right| \\
& \leq \epsilon \cdot \rho\left(x_{1}, y_{1}\right)+\ldots+\epsilon \cdot \rho\left(x_{n}, y_{n}\right) \\
& \leq \epsilon a .
\end{aligned}
$$

Since this holds for all $a$ with $\tilde{\rho}_{3}(x, y)<a<\delta$, we conclude that $\mid \tilde{f}([x])-$ $\tilde{f}([y]) \mid \leq \epsilon \cdot \tilde{\rho}_{3}(x, y)$. Thus we have shown that $\tilde{\rho}_{3}(x, y)<\delta$ implies $|\Phi \tilde{f}([x],[y])| \leq \epsilon ;$ so $\tilde{f} \in \operatorname{lip}(\tilde{X})$.

Lemma 3.3. Let $X$ be a compact metric space and suppose $\mathcal{A} \leq \operatorname{lip}(X)$ separates points. Let $Y$ be the set $X$ with the metric $\rho_{\mathcal{A}}$. Then $\mathcal{A}$ is isometrically contained in $\operatorname{lip}(Y)$.

Proof. Let $f \in \mathcal{A}$ and let $f^{\prime}$ denote the same map with domain $Y$. Then obviously $\|f\|_{\infty}=\left\|f^{\prime}\right\|_{\infty} ; L(f) \leq L\left(f^{\prime}\right)$ since the identity map from $X$ to $Y$ is nonexpansive; and $L(f) \geq L\left(f^{\prime}\right)$ by the definition of $\rho_{\mathcal{A}}$. So $\|f\|_{L}=\left\|f^{\prime}\right\|_{L}$. This shows that $\mathcal{A}$ is isometrically contained in $\operatorname{Lip}(Y)$.

Now we must show $\mathcal{A} \subset \operatorname{lip}(Y)$. Let $f \in \mathcal{A}$; we may assume $\|f\|_{L}=1$. Let $0<\epsilon<1$.

Find $0<\delta<1$ such that $\rho(x, y) \leq \delta$ implies $|f(x)-f(y)| \leq \epsilon \cdot \rho(x, y)$. We claim that there exists $\delta^{\prime}>0$ such that $\rho_{\mathcal{A}}(x, y) \leq \delta^{\prime}$ implies $\rho(x, y) \leq \epsilon \delta$. For if not then we could find a sequence $\left(x_{n}, y_{n}\right) \subset X^{2}$ such that $\rho\left(x_{n}, y_{n}\right)>\epsilon \delta$ for all $n$ and $\rho_{\mathcal{A}}\left(x_{n}, y_{n}\right) \rightarrow 0$. Taking a convergent subsequence $\left(x_{n_{k}}, y_{n_{k}}\right) \rightarrow$ $(x, y)$, we get $\rho_{\mathcal{A}}(x, y)=0$ and $\rho(x, y) \neq 0$, which contradicts the assumption that $\mathcal{A}$ separates points.

We now claim that for any $x, y \in X, \rho_{\mathcal{A}}(x, y) \leq \delta^{\prime}$ implies $|f(x)-f(y)| \leq$ $\epsilon \cdot \rho_{\mathcal{A}}(x, y)$; this will establish that $f \in \operatorname{lip}(Y)$ and complete the proof. To verify this claim, suppose $x, y \in X$ satisfy $\rho_{\mathcal{A}}(x, y) \leq \delta^{\prime}$. Then

$$
|f(x)-f(y)| \leq \rho(x, y) \leq \epsilon \delta .
$$

Without loss of generality suppose $f(x) \geq f(y)$ and define

$$
g=[(f \wedge f(x)) \vee f(y)]-f(y)
$$

this is in $\mathcal{A}$ by Proposition 1.2. Note that

$$
g(z)= \begin{cases}0 & \text { if } g(z)<f(y) \\ g(z)-f(y) & \text { if } f(y) \leq g(z) \leq f(x) \\ f(x)-f(y) & \text { if } g(z)>f(x)\end{cases}
$$


Now for any $x^{\prime}, y^{\prime} \in X$, if $\rho\left(x^{\prime}, y^{\prime}\right) \leq \delta$ then

$$
\left|\Phi g\left(x^{\prime}, y^{\prime}\right)\right| \leq\left|\Phi f\left(x^{\prime}, y^{\prime}\right)\right| \leq \epsilon
$$

and if $\rho\left(x^{\prime}, y^{\prime}\right) \geq \delta$ then

$$
\left|g\left(x^{\prime}\right)-g\left(y^{\prime}\right)\right| \leq|f(x)-f(y)| \leq \epsilon \delta \leq \epsilon \cdot \rho\left(x^{\prime}, y^{\prime}\right),
$$

hence again $\left|\Phi g\left(x^{\prime}, y^{\prime}\right)\right| \leq \epsilon$. So $L(g) \leq \epsilon$, and also

$$
\|g\|_{\infty}=f(x)-f(y) \leq \epsilon \delta \leq \epsilon .
$$

Thus $\|g\|_{L} \leq \epsilon$, so we get

$$
\rho_{\mathcal{A}}(x, y) \geq|g(x)-g(y)| / \epsilon=|f(x)-f(y)| / \epsilon,
$$

as desired.

Theorem 3.4. Let $X$ be a compact metric space, let $\mathcal{A} \leq \operatorname{lip}(X)$, and let $\pi: X \rightarrow X_{\mathcal{A}}$ be the natural projection. Then $T_{\pi}: f \mapsto f \circ \pi$ takes $\operatorname{lip}\left(X_{\mathcal{A}}\right)$ isometrically onto $\mathcal{A}$.

Proof. Set $x \sim y$ iff $f(x)=f(y)$ for all $f \in \mathcal{A}$ and let $\tilde{X}$ be the metric quotient of $X$ by $\sim$. Then $\tilde{X}$ and $X_{\mathcal{A}}$ have the same underlying set (i.e. in this case the equivalence relation determined by $\tilde{\rho}_{1}$ coincides with $\sim$ ) and we may also consider $\pi$ as a map from $X$ onto $\tilde{X}$. Since every function in $\mathcal{A}$ respects $\sim$, Proposition 2.2 implies that $\mathcal{A} \subset T_{\pi}(\operatorname{Lip}(\tilde{X}))$. Since $T_{\pi}: \operatorname{Lip}(\tilde{X}) \rightarrow \operatorname{Lip}(X)$ is an isometry by Lemma 3.1 it therefore suffices to show that $T_{\pi}^{-1}(\mathcal{A}) \subset \operatorname{Lip}(\tilde{X})$ is isometrically isomorphic to $\operatorname{lip}\left(X_{\mathcal{A}}\right)$ (via the identity map).

By Lemma $3.2, T_{\pi}^{-1}(\mathcal{A}) \subset \operatorname{lip}(\tilde{X})$, so that (with $T_{\pi}^{-1}(\mathcal{A})$ in place of $\mathcal{A}$ and $\tilde{X}$ in place of $X)$ this reduces us to the case where $\mathcal{A}$ separates points and $\pi: X \rightarrow X_{\mathcal{A}}$ is the identity. In this case Lemma 3.3 shows that $\mathcal{A} \subset \operatorname{lip}\left(X_{\mathcal{A}}\right)$ isometrically. It is clear from the definition of $\rho_{\mathcal{A}}$ that $\mathcal{A}$ has the separation property for $X_{\mathcal{A}}$ for any $a>1$, so we finally get $\mathcal{A}=\operatorname{lip}\left(X_{\mathcal{A}}\right)$ by the pseudo Stone-Weierstrass theorem.

\section{References}

[BCD] W.G.Bade, P.C. Curtis, Jr., and H.G. Dales, Amenability and weak amenability for Beurling and Lipschitz algebras, Proc. Lond. Math. Soc., 55 (1987), 359-377.

[Ha] L.G. Hanin, Kantorovich-Rubinstein norm and its application in the theory of Lipschitz spaces, Proc. Amer. Math. Soc., 115 (1992), 345-352. 
[He] L.I. Hedberg, The Stone-Weierstrass theorem in Lipschitz algebras, Ark. Mat., 8 (1969), 63-72.

[Mc] E.J. McShane, Extension of range of functions, Bull. Amer. Math. Soc., 40 (1934), 837-842.

[Mi] H. Mirkil, Continuous translation of Hölder and Lipschitz functions, Canad. J. Math., 12 (1960), 674-685.

[Sh] D.R. Sherbert, The structure of ideals and point derivations in Banach algebras of Lipschitz functions, Trans. Amer. Math. Soc., 111 (1964), 240-272.

[St] M.H. Stone, The generalized Weierstrass approximation theorem, Math. Mag., 21 (1948), 167-184 and 237-254.

[W1] N. Weaver, Duality for locally compact Lipschitz spaces, to appear in Rocky Mtn J. Math.

[W2] , Lattices of Lipschitz functions, Pacific J. Math., 164 (1994), 179-193.

Received August 1, 1993 and revised May 1, 1994.

UNiversity of California

SANTA BARbara, CA 93106

E-mail address: weaver@math.ucsb.edu 



\title{
PACIFIC JOURNAL OF MATHEMATICS
}

Founded by

\author{
$\begin{array}{ll}\text { E. F. Beckenbach (1906-1982) F. Wolf (1904-1989) } & \text { F }\end{array}$
}

EDITORS

Sun-Yung A. Chang (Managing Editor) Robert Finn

University of California

Los Angeles, CA 90095-1555

pacific@math.ucla.edu

F. Michael Christ

University of California

Los Angeles, CA 90095-1555

christ@math.ucla.edu

Nicholas Ercolani

University of Arizona

Tucson, AZ 85721

ercolani@math.arizona.edu
Stanford University

Stanford, CA 94305

finn@gauss.stanford.edu

Steven Kerckhoff

Stanford University

Stanford, CA 94305

spk@gauss.stanford.edu

Martin Scharlemann

University of California

Santa Barbara, CA 93106

mgscharl@math.ucsb.edu
Gang Tian

Massachusettes Institute of Technology

Cambridge, MA 02139

tian@math.mit.edu

\section{S. Varadarajan}

University of California

Los Angeles, CA 90095-1555

vsv@math.ucla.edu

Dan Voiculescu

University of California

Berkeley, CA 94720

dvv@math.berkeley.edu

\section{SUPPORTING INSTITUTIONS}

\author{
CALIFORNIA INSTITUTE OF TECHNOLOGY \\ NEW MEXICO STATE UNIVERSITY \\ OREGON STATE UNIVERSITY \\ STANFORD UNIVERSITY \\ UNIVERSITY OF ARIZONA \\ UNIVERSITY OF BRITISH COLUMBIA \\ UNIVERSITY OF CALIFORNIA \\ UNIVERSITY OF HAWAII
}

\author{
UNIVERSITY OF MONTANA \\ UNIVERSITY OF NEVADA, RENO \\ UNIVERSITY OF OREGON \\ UNIVERSITY OF SOUTHERN CALIFORNIA \\ UNIVERSITY OF UTAH \\ UNIVERSITY OF WASHINGTON \\ WASHINGTON STATE UNIVERSITY
}

The supporting Institutions listed above contribute to the cost of publication of this Journal, but they are not owners or publishers and have no responsibility for its contents or policies.

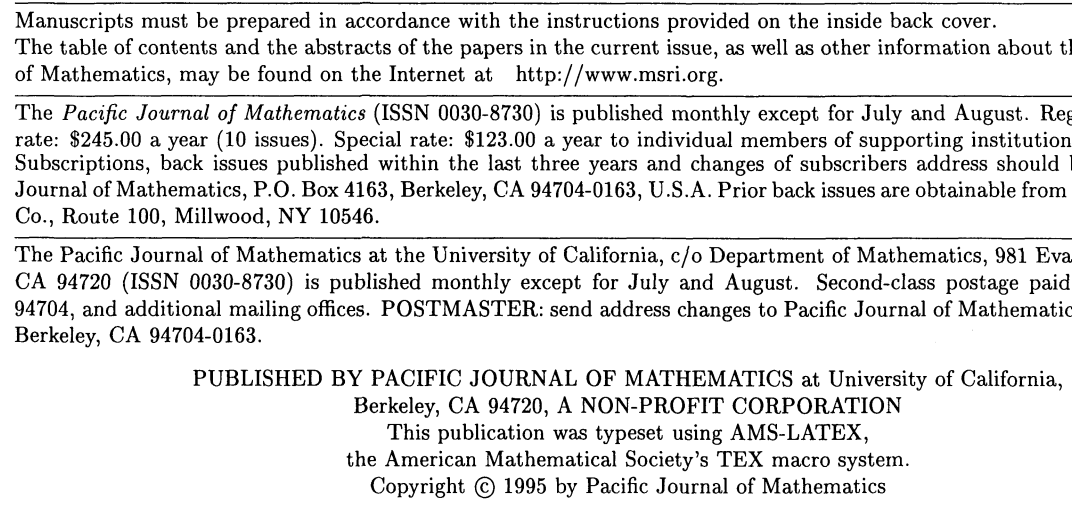




\section{PACIFIC JOURNAL OF MATHEMATICS}

\section{Volume $173 \quad$ No. $1 \quad$ March 1996}

Isometric immersions of $H_{1}^{n}$ into $H_{1}^{n+1}$

KINETSU ABE

Rotationally symmetric hypersurfaces with prescribed mean curvature

MARIE-FRANÇOISE BIDAUT-VÉRON

The covers of a Noetherian module

JIAN-JUN CHUAI

On the odd primary cohomology of higher projective planes

MARK FoSKEY and MiCHAEL DAVID SLACK

Unit indices of some imaginary composite quadratic fields. II

MiKinito HiRABAYASHI

Mixed automorphic vector bundles on Shimura varieties

MIN Ho LeE

Trace ideal criteria for Toeplitz and Hankel operators on the weighted Bergman

spaces with exponential type weights

PENG LIN and RICHARD ROCHBERG

On quadratic reciprocity over function fields

KATHY DONOVAN MERRILL and LYNNE WALLING

$\left(A_{2}\right)$-conditions and Carleson inequalities in Bergman spaces

TAKAHIKO NAKAZI and MASAHIRO YAMADA

A note on a paper of E. Boasso and A. Larotonda: "A spectral theory for solvable Lie 173 algebras of operators"
C. Отт

Tensor products with anisotropic principal series representations of free groups

Carlo Pensavalle and Tim Steger

On Ricci deformation of a Riemannian metric on manifold with boundary

YING SHEN

The Weyl quantization of Poisson $S U(2)$

Albert Jeu-Liang Sheu

Weyl's law for $S L(3, \mathbb{Z}) \backslash S L(3, \mathbb{R}) / S O(3, \mathbb{R})$

ERIC GeORge Stade and Dorothy IRENe Wallace (ANDREOli)

Minimal hyperspheres in two-point homogeneous spaces

PER TOMTER

Subalgebras of little Lipschitz algebras

NiKOLAI ISAAC WEAVER 